\title{
David ANDRESS (dir.), Experiencing the French
}

\section{Revolution}

Oxford, Voltaire Foundation, University of Oxford, 2013

Annie Jourdan

\section{(2) OpenEdition \\ Journals}

Édition électronique

URL : https://journals.openedition.org/ahrf/13660

DOI : 10.4000/ahrf.13660

ISSN : 1952-403X

Éditeur :

Armand Colin, Société des études robespierristes

Édition imprimée

Date de publication : 1 septembre 2015

Pagination : 256-258

ISBN : 9782200929855

ISSN : 0003-4436

Référence électronique

Annie Jourdan, «David Andress (dir.), Experiencing the French Revolution », Annales historiques de la Révolution française [En ligne], 381 | juillet-septembre 2015, mis en ligne le 08 janvier 2016, consulté le 22 avril 2022. URL : http://journals.openedition.org/ahrf/13660; DOI : https://doi.org/10.4000/ahrf 13660

Ce document a été généré automatiquement le 22 avril 2022.

Tous droits réservés 


\title{
David ANDRESS (dir.), Experiencing the French Revolution
}

Oxford, Voltaire Foundation, University of Oxford, 2013

\author{
Annie Jourdan
}

\section{RÉFÉRENCE}

David ANDRESS (dir.), Experiencing the French Revolution, Oxford, Voltaire Foundation, University of Oxford, 2013, 332 p., ISSN 0435-2866, $65 £ / 85 €$.

1 Le livre, édité par la Fondation Voltaire, est le résultat des débats, tenus en 2010, lors du colloque organisé par l'Université de Portsmouth. Le colloque lui-même réunissait une pléiade d'historiens français, anglais, australiens, canadiens et américains, tous spécialistes de la Révolution française. L'objectif était de réfléchir sur les débats récents autour de la dite révolution. L'initiative poursuivait en vérité celle initiée par les French Historical Studies (vol. 32, 2009) qui avaient publié un gros dossier sur l'historiographie révolutionnaire depuis le bicentenaire et celle de $\mathrm{H}$-France qui y avait consacré un $\mathrm{H}$ France Salon. Dans le recueil des actes, David Andress a curieusement exclu les contributions françaises. On y trouvera donc seulement des articles d'Anglophones. Cela ne préjuge en aucun cas de la qualité des uns et des autres, mais cela vaut la peine d'être noté.

2 Le titre annonce la couleur. S'inspirant de propos de Lynn Hunt (FHS, 2009), Andress s'interroge sur ce que les sciences peuvent apporter à l'historien, pour ce qui est des expériences vécues et des émotions ressenties par les révolutionnaires. C'est pour conclure qu'en réalité, les archives et les sources sont incapables de nous fournir les détails nécessaires pour redécouvrir ce que ces hommes et femmes ont ressenti sur le moment. Faute d'informations fiables, mieux vaut étudier les interactions des individus avec le monde et les réalités qui les entourent. Mieux vaut explorer les diverses appréhensions des circonstances par les acteurs qui les vivent et les témoignages qu'ils nous en laissent. Le fait est - nous semble-t-il - que les expériences vécues par les 
révolutionnaires ont provoqué chez eux tout à la fois de grandes attentes et de terribles frustrations. Depuis 1787 et la première Assemblée des notables, et, bien au-delà de la prise de la Bastille, ce sont ces deux sentiments qui ont prévalu et qui sont à l'origine de bien des drames.

Le livre est partagé en trois parties, plus ou moins cohérentes ainsi qu'il en va souvent avec les recueils d'actes. Sous le titre de "Experiencing revolutionary transitions », la première se concentre sur les grandes tendances culturelles et les trajectoires individuelles. Dans un article sérieusement informé et chiffré, Simon Burrows reconsidère les thèses de Robert Darnton sur la littérature clandestine qui pénètre bon gré mal gré dans le royaume de France. En se concentrant exclusivement sur les productions de la Société typographique de Neuchâtel, il conclut un peu rapidement que la Révolution française n'a pas été provoquée par les livres subversifs. Un peu rapidement car c'est oublier qu'entre-temps, Loménie de Brienne avait permis aux Français de s'exprimer sur les États-généraux et, donc, sur les sujets politiques. Les années 1788-1789 voient ainsi une myriade de publications s'amonceler dans les boutiques et cabinets de lecture. Si l'on en croit Arthur Young, La RochefoucauldLiancourt les collectionnait. Ses archives privées devraient pourvoir donner une idée de tout ce qui s'est publié à l'époque. Des recherches qui s'imposent, me semble-t-il. Ces productions mériteraient elles-mêmes d'être recensées, comptées, évaluées. En vérité, on en trouve dès 1786. Qui plus est, le gouvernement lui-même laissait bien imprudemment publier des ouvrages "révolutionnaires", tels que la traduction des constitutions américaines que demanda Benjamin Franklin à Vergennes, en 1783. Cela dit, le travail effectué par Burrows et ses collaborateurs est d'une grande valeur, mais les résultats se limitant à la Société typographique de Neuchâtel, les questionnements demeurent.

Originale est la contribution de Charles Walton sur les dons patriotiques, leurs motivations et implications. L'auteur découvre ainsi que le don joue un rôle important durant les années révolutionnaires, à tel point qu'il valorise et protège ceux qui ont su se montrer généreux. Deux des fermiers généraux seront ainsi épargnés sous la Terreur, en raison de leur générosité passée ou récente (p.65). Vient ensuite Peter McPhee qui traite de Robespierre et de la violence. Le sujet est moins novateur. Tout historien de la Révolution sait fort bien que Robespierre était un adversaire de la peine de mort, avant de s'en faire quelques années plus tard le zélé défenseur et de passer dans l'histoire comme l'incarnation de la Terreur. Sur ce point aussi, des recherches nouvelles devraient être entreprises, notamment sur les renvois au gouvernement révolutionnaire, exigés par le Bureau de Police générale et signés par Robespierre ou Saint-Just. Les travaux d'Arne Örding devraient être revus et corrigés. Mette Harder, à qui l'on doit une belle thèse sur la Terreur conventionnelle et les purges parlementaires de 1793-1799 - qui va bientôt paraître - propose un portrait de Tallien, symbole même de la girouette et de l'opportunisme de l'époque, qui met à profit ses expériences pour se tirer de tout mauvais pas. En 1815, il est un des rares conventionnels à ne pas être exilé par Louis XVIII. Harder se demande pourquoi. Il semblerait que ce soit parce qu'entre-temps, il était devenu infirme - et aveugle. (Sur la Restauration, Mette Harder aurait pu consulter dans la série $\mathrm{F}^{7}$ des Archives nationales les dossiers de Tallien : $\mathrm{F}^{7} 6683$ et $\mathrm{F}^{7}$ 6715). Cette cécité en rappelle une autre : celle d'Æ̇dipe, rongé par le remords. Se pourrait-il que ce fût le cas de Tallien? Se pourrait-il que ce soit le symptôme d'une mauvaise conscience, héritée justement de ses expériences et ses actes? Harder ne le dit pas. Autre contribution passionnante, celle 
d'Ian Germani sur la justice militaire dans les armées révolutionnaires. Germani démontre, archives à l'appui, que cette justice sut marier rigueur et tolérance et nuance donc les études qui mettent trop l'accent sur une justice terroriste, meurtrière et aveugle.

La deuxième partie, intitulée "Experiences at the heart of the Terro ", rassemble les articles d'Alex Fairfax-Cholmeley, Jonathan Smyth, Ronen Steinberg et Marisa Linton. Ces quatre articles sont excellents. Le plus novateur est sans nul doute celui de FairfaxCholmeley qui étudie les pétitions et écrits des victimes de la Terreur, soucieux de se disculper et de retrouver la confiance des autorités ou des sections. Cette littérature nommée ici « contre-dénonciation » devrait donc être prise en compte dans toute étude de la Terreur, car ses auteurs étaient, qui plus est, soutenus par des tiers. Quels en ont été au juste les effets? Jonathan Smyth, lui, analyse la réception dans plusieurs départements de la fête de l'Être suprême, avant de conclure qu'elle fut bien accueillie. Il conteste qu'elle ait été vue comme un événement "stérile", ainsi qu'on a trop tendance à le dire. Ronen Steinberg, dont les recherches actuelles portent sur les héritages de la Terreur, aborde les effets de la période sur les acteurs et en étudie les traumatismes. Pour ce faire, il étudie des textes politiques et médicaux. Peut-être pourrait-il y inclure aussi les œuvres d'art exposées dans les salons du Directoire, qui en disent long justement sur ces traumatismes. Toutefois, il entre vraiment dans le vif du sujet: l'expérience révolutionnaire. Autre expérience: celle qu'interroge Marisa Linton et qui concerne les tentatives d'assassinat dont sont victimes plusieurs conventionnels. Linton note bien qu'en dépit de la rhétorique propre au discours politique, ces tentatives sont réelles et prises au sérieux par les conventionnels. Après tout, Lepelletier et Marat ont bel et bien été assassinés. Robespierre se sent lui-même menacé. Ce qui lui permet de se donner et de passer pour l'homme vertueux par excellence, tout à la fois martyr et héros - sur un ton très particulier par rapport notamment à Madame Roland. La dernière partie évoque quelques expériences révolutionnaires étrangères. Brecht Deseure consacre une étude passionnante aux discours des Français en Belgique. Or ces discours tout politiques dévoilent avant tout un souci de persuader en douceur les nouveaux Français de la continuité entre l'histoire belge et la Révolution française. Les fonctionnaires sont particulièrement doués pour invoquer les libertés "belgiques", dont ils tirent des exemples dans l'histoire du cru. Ce faisant, Deseure nuance ce qu'écrivait François Furet à propos de la rupture temporelle et du rejet de l'histoire qu'aurait imposés la Révolution. Enfin, Ffion Jones donne un aperçu de l'impact français dans le pays de Galles, qui, avec l'Irlande, a été le seul territoire britannique, envahi un temps par l'armée française et qui a peu attiré l'attention jusqu'ici. L'expérience fut brisée par la répression anglaise - tout comme en Irlande. Le volume se clôt sur une contribution de J. Ward Regan qui traite $\mathrm{du}$ premier journaliste révolutionnaire, d'envergure internationale, Thomas Paine et des conséquences de son engagement en France, dont témoignent ses écrits ultérieurs. Il en est d'autres, du reste, tels Joel Barlow, un poète américain qui se politise au rythme des aléas de la Révolution française et de ses priorités propres, ou Gilbert Imlay, l'amant de Mary Wollstonecraft, elle-même très politisée.

$6 \quad$ En bref, tous les textes publiés ici nuancent les interprétations dites révisionnistes. Ils proposent des points de vue originaux et des angles d'approche qui ne le sont pas moins. Plusieurs le font à partir de sources négligées. Tous, d'une façon ou d'une autre, abordent le difficile problème de l'expérience en temps de révolution. Et ainsi que l'annonce et le souhaite David Andress, ils montrent bien la multiplicité de perspectives 
qu'offre le thème même d'expérience, et combien il est préjudiciable d'en négliger l'étude au profit du seul discours. 